\title{
Who Cares? Women with Breast Cancer and Their Significant Other
}

\section{Ashling Rosanna Murphy}

\author{
School of Nursing \& Midwifery, UCC
}

"No one is useless in this world who lightens the burdens of another." Charles Dickens

\section{Introduction}

The news comes. What was feared is now a reality. Devastation. The words "you", "breast" and "cancer" echo throughout the room. What happens now? Who do you turn to? Nearly 3,000 women are diagnosed with breast cancer in Ireland on an annual basis. The above scenario for them is very much a reality. A diagnosis of cancer is devastating. It is not only the disease itself but the treatments, life alteration and in some cases acceptance of defeat that the person endures. With cancer it is rarely one individual who suffers. With breast cancer (one of the most commonly diagnosed cancers in Ireland) an invasion on the woman's identity can occur, bringing great distress. The people who support women with breast cancer throughout the diagnosis and disease play a vital part in the maintenance of the woman's wellbeing. It is human nature to seek out comfort from other people. The main area of support that a woman will receive comes from a familiar person, someone close to the woman who already had a relationship with her, prior to the diagnosis of breast cancer. The relationship between women with breast cancer and their significant other is highly beneficial to their care. The significant other is predominantly the partner or spouse of the woman however, others such as siblings, mother, child or friend have been identified as being significant others for women with breast cancer. This is specifically true in situations where the woman may be unmarried/separated or their partner may be deceased. Regardless of whether it is a spouse or other significant person, this relationship acts as a support structure throughout the cancer journey. It supports the psychological, emotional and physical aspects of the woman's life. This paper is a discussion on the importance of the relationship between women with breast cancer and their significant other. It provides the background for a PhD study that will examine this relationship and its potential implications on the health of the woman with breast cancer and her significant other. 


\section{Background}

Breast cancer is recognised globally as one of the most common cancers. Described as a chronic disease, breast cancer poses great stress and challenges to the individual and their loved ones. Often breast cancer can result in physical and/or psychological problems that the woman may seek support for. Within the current healthcare system the majority of continued care to women with breast cancer is provided by family and friends. This is due to care being transferred from acute hospitals to outpatient clinics. As a result of this, the process of informal care, care provided by family and friends, is increasing within the breast cancer context. It is estimated that over half of all care is provided by informal carers. On an annual basis it is estimated that informal carers save the health service in Ireland two billion euro.

With increasing pressure on the health service the process of informal caring has become increasingly prevalent. This support includes physical assistance with the management of side effects, emotional support, financial help and spiritual guidance. Women with breast cancer have indicated the importance of having a close individual involved in their care. Significant others who are involved in the care of woman with breast cancer can promote positive outcomes. Supportive relationships have been linked with lower levels of depression, anxiety and stress. Exploring the relationship between women with breast cancer and their significant other may provide reasons as to why these outcomes are positive.

\section{The Benefits of Relationships between Women and Their Significant Other}

Relationships and their quality have been seen to significantly impact on women with breast cancer. It has been recognized for several years that social support in breast cancer is an important factor which may affect the general well-being of those diagnosed with cancer. Relationships can affect the woman's adjustment to the breast cancer and the woman's overall health outcomes. Women diagnosed with breast cancer who have supportive, communicative and involved significant others are seen to exhibit better adjustment to the breast cancer. The support and connectedness provided by another assists women in dealing with the breast cancer. The fact that research focusing on the relationship between women with breast cancer and their significant other has not been conducted previously provides an interesting and enlightening focus for my $\mathrm{PhD}$.

A breast cancer diagnosis not only affects the woman diagnosed but also has huge implications for those involved in their life. The diagnosis can alter the existing relationship that was present. Side effects of the breast cancer treatment including fatigue, lethargy, hair 
loss and nausea can all affect the woman's relationship with their significant other. This can be detrimental to the woman's prognosis as women with breast cancer have identified the strong need to maintain close relationships with their significant other. The significant others of women with breast cancer have also stated the importance of the maintenance of the relationship.

The concept of connectedness is strongly linked with the quality of the relationship that the women with breast cancer and their significant other experience. Feeling close to someone throughout the breast cancer journey is essential to the wellbeing of women. It is also important to maintain the relationship and while change is inevitable in breast cancer, the extent of the effect that the breast cancer has on the relationship is a research area that is ripe for investigation. Results of the study could enhance care for women with breast cancer and their loved ones.

\section{Methodology}

The lack of available studies specifically focusing on the woman with breast cancer and their significant other has prompted this study. The possible outcomes could be seen as beneficial to identifying support networks for women with breast cancer and as suggested above may impact on healthcare costs. Future focus on the mechanisms of the relationship between the woman with breast cancer and their significant other needs to be considered.

A quantitative study will be conducted using validated questionnaires. The components being measured are relationship orientation (type of relationship), relationship behaviours (this relates to how the individuals in the relationship act towards each other whether supportive or dismissive), relationship mediators (responsiveness to breast cancer diagnosis and disease) and relationship outcomes (satisfaction with relationship and commitment to each other) and health and disease outcomes. The questionnaires will be distributed to both women with breast cancer and their identified significant other. This is to ensure that both perspectives of the relationship and health outcomes are obtained. The women will be asked to identify who they see as their significant other, this is to ensure that it is the woman's view that is taken into consideration and not the researchers. A number of questionnaires will be used to ensure that each relevant area of the relationship is investigated. The questionnaires will measure each of the variables above. By measuring several dimensions we can gain an overall view of the relationship and its impact on health outcomes. 


\section{Aspects of the Relationship}

Relationships orientation involves the attachment style of the individuals involved in the relationship. This is how well one individual bonds with the other. There are four relationship styles or orientations. These are secure, avoidant, ambivalent or dismissive. Assessing the type of relationship that the woman with breast cancer and her significant other has is beneficial, as this relationship style affects how well the woman and her significant other communicate with each other. For example women who have secure relationship styles will be more open and secure in their relationship. Women who have avoidant relationship styles will disengage from communication and avoid difficult situations in the relationship. Those with an ambivalent relationship style will be disingenuous in their relationship. Individuals with dismissive relationship styles will be unsupportive in the relationship and provide little security.

Relationship behaviours involve the acts within the relationship such as support, caregiving and social negativity. Support is the provision of aid to another. This is important to assess in this study as support is one of the highest ranked needs that women with breast cancer have. The need for the significant other of the woman to feel supported is also important and for this reason support in terms of what the woman receives and what the significant others receives will both be assessed. Caregiving can involve all the daily acts of providing assistance with physical needs as well as emotional needs such as listening and comforting. Social negativity involves the negative or confrontational aspects of the relationship such as arguing, ignoring etc. These need to be assessed as negative behaviours within the relationship may affect the well-being of those involved in the relationship. Individuals who are involved in argumentative and confrontational relationships will have poorer coping strategies and as a result poorer outcomes both in terms of the relationship and in their overall health.

Those items that mediate the relationship such as partner involvement and responsiveness are important. How well a partner or significant other deals with the diagnosis of cancer and responds to treatments or side effects can influence how well the woman diagnosed copes with them also. The relationship outcomes regarding relationship satisfaction and commitment are determinants of the health of the woman and their significant other. Individuals with higher satisfaction in their relationship and a greater sense of commitment will cope better with breast cancer and endeavour to overcome the challenges that breast cancer brings. After assessing the above criteria in terms of the relationship, the health status of the woman and their significant other will be assessed using a scale to measure quality of life. The results will indicate whether relationship style, behaviours, mediators and outcomes influence the quality of lives of women with breast cancer and their significant other. 


\section{Conclusion}

The relationship between women with breast cancer and their significant other(s) is inherently complex and multidimensional. The process of this relationship works on the basis that when one party is unwell the other provides the necessary act of caring. The relationship does not flow in one direction rather it is haphazard in nature, continually changing to meet the demands of those involved in the relationship. In breast cancer the quality of life, psychological, emotional and physical aspects of the woman are significantly enhanced when care is provided by a familiar person. However, the physical and mental burden that caring can place on the woman's significant other can impact on both people in the relationship. The relationship can also impact on the adjustment to breast cancer. Research that explores and contributes to the understanding of relationships among women with breast cancer may enhance knowledge around the relationship process and its impact on outcomes for these women. The primary function of research is to provide an insight into the understanding of impacting factors with an aim to improve outcomes. Women's significant others are already providing support (both physical and emotional) and comfort as well as attempting to remain in the role they had prior to the breast cancer diagnosis. Although the connections between relationships and health are well established, less is known about the interpersonal processes through which relationships influence health outcomes. Exploring the relationship between women with breast cancer and their significant other(s) may provide insight into the act of caring within a relationship context. An insight into the nature of caring relationships women with breast cancer experience would enhance healthcare professionals understanding of the crucial role that women's significant others play throughout their breast cancer journey.

With appreciation and gratitude to my supervisors Professor Josephine Hegarty, Dr. Mairin O Mahony, Dr.Mark Corrigan and Dr. Suzanne Denieffe for their support. The Ashling Rosanna Murphy is in receipt of the UCC Strategic Research Fund. 